\title{
Enabling indoor localization with internet of things (IoT)
}

\begin{abstract}
This work presents an indoor localization service with the aid of received signal strength indicator (RSSI) from IoT. The RSSI emmitted from the IoT are converted into the relative distance between the IoT nodes to determine the current location. In order to enhance the accuracy, the area of interest is further divided into few zones. The RSSI from the nearby IoT devices (BLE Beacons) detected in each zones are collected. This enable the system to first identify the correct zone located, and utilizing the relative distance from the RSSI at the zone to further acquire the current position inside the zone. A demo system has been developed with the Bluetoothe Low Energy (BLE) to evaluate the indoor localization service with this zone system.
\end{abstract}

Keyword: Indoor positioning system; Bluetooth low energy (BLE); Received signal strength indicator (RSSI); Relative distance; Zones; IOT devices; Practical approach; Low cost solution; Low power devices; CC2541; CR2032 